\title{
Influence of non-local thermodynamic equilibrium and Zeeman effects on magnetic equilibrium reconstruction using spectral motional Stark effect diagnostic
}

R. Reimer, , O. Marchuk, , B. Geiger, , P. J. Mc Carthy, , M. Dunne, , J. Hobirk, , R. Wolf, and, and

Citation: Review of Scientific Instruments 88, 083509 (2017); doi: 10.1063/1.4994889

View online: http://dx.doi.org/10.1063/1.4994889

View Table of Contents: http://aip.scitation.org/toc/rsi/88/8

Published by the American Institute of Physics

\section{Articles you may be interested in}

Development of beam emission spectroscopy diagnostic on EAST

Review of Scientific Instruments 88, 083505 (2017); 10.1063/1.4997074

A new high sensitivity far-infrared laser interferometer for the HL-2A tokamak

Review of Scientific Instruments 88, 083508 (2017); 10.1063/1.4997070

Correlation electron cyclotron emission diagnostic in TCV

Review of Scientific Instruments 88, 083506 (2017); 10.1063/1.4997075

Measurement of the velocity of neutral fragments by the "correlated ion and neutral time of flight" method combined with "velocity-map imaging"

Review of Scientific Instruments 88, 083101 (2017); 10.1063/1.4991828

A high voltage pulse generator based on silicon-controlled rectifier for field-reversed configuration experiment Review of Scientific Instruments 88, 083507 (2017); 10.1063/1.4997077

Enhanced fast digital integrator for magnetic measurements

Review of Scientific Instruments 88, 085103 (2017); 10.1063/1.4996539

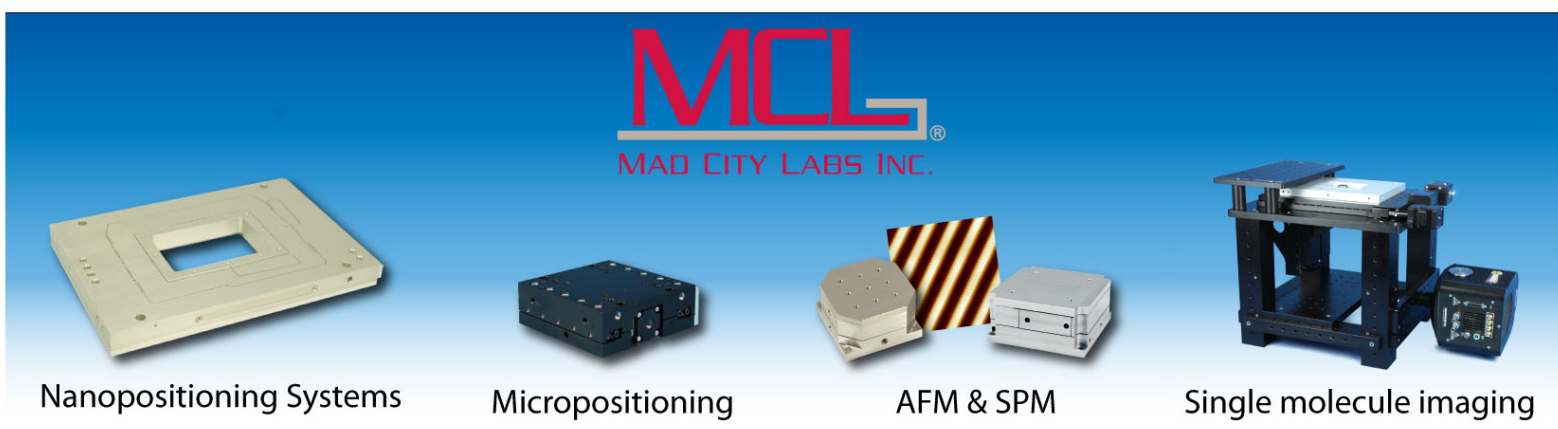




\title{
Influence of non-local thermodynamic equilibrium and Zeeman effects on magnetic equilibrium reconstruction using spectral motional Stark effect diagnostic
}

\author{
R. Reimer, ${ }^{1}$ O. Marchuk, ${ }^{2}$ B. Geiger, ${ }^{3}$ P. J. Mc Carthy, ${ }^{4}$ M. Dunne, ${ }^{3}$ J. Hobirk, ${ }^{3}$ R. Wolf, ${ }^{1}$ \\ and ASDEX Upgrade Team ${ }^{3, a)}$ \\ ${ }^{1}$ Max-Planck-Institut für Plasmaphysik, EURATOM Association, Teilinstitut Greifswald, Wendelsteinstraße 1, \\ 17491 Greifswald, Germany \\ ${ }^{2}$ Institut für Energie und Klimaforschung-Plasmaphysik, Forschungszentrum Juelich GmbH, \\ 52425 Jülich, Germany \\ ${ }^{3}$ Max-Planck-Institut für Plasmaphysik, EURATOM Association, Boltzmannstraße 2, 85748 Garching, Germany \\ ${ }^{4}$ Department of Physics, University College Cork, Association EURATOM-DCU, Cork, Ireland
}

(Received 13 February 2017; accepted 3 July 2017; published online 9 August 2017)

The Motional Stark Effect (MSE) diagnostic is a well established technique to infer the local internal magnetic field in fusion plasmas. In this paper, the existing forward model which describes the MSE data is extended by the Zeeman effect, fine-structure, and relativistic corrections in the interpretation of the MSE spectra for different experimental conditions at the tokamak ASDEX Upgrade. The contribution of the non-Local Thermodynamic Equilibrium (non-LTE) populations among the magnetic sub-levels and the Zeeman effect on the derived plasma parameters is different. The obtained pitch angle is changed by $3^{\circ} \ldots 4^{\circ}$ and by $0.5^{\circ} \ldots 1^{\circ}$ including the non-LTE and the Zeeman effects into the standard statistical MSE model. The total correction is about $4^{\circ}$. Moreover, the variation of the magnetic field strength is significantly changed by $2.2 \%$ due to the Zeeman effect only. While the data on the derived pitch angle still could not be tested against the other diagnostics, the results from an equilibrium reconstruction solver confirm the obtained values for magnetic field strength. [http://dx.doi.org/10.1063/1.4994889]

\section{INTRODUCTION}

The accurate measurements of the local magnetic field are a quite demanding task in fusion plasmas, and the Motional Stark Effect (MSE) diagnostic represents probably the most sensitive and suitable instrument to deliver the necessary information. In general, the MSE concept relies on the observation of the Balmer- $\alpha$ transition $(n=2 \rightarrow 3)$ emitted from injected high energetic $(10 \ldots 100 \mathrm{keV} / \mathrm{u})$ deuterium or hydrogen particles with velocity $\vec{v}$ excited by collisions with plasma ions and electrons. The plasma is confined by the background magnetic field on the order of $1 \ldots 5 \mathrm{~T}$. The observed emission is split into the nine observable Stark components by the Lorentz electric field $\vec{E}_{L}, \vec{E}_{L}=\vec{v} \times \vec{B}$, acting on atoms in their co-moving frame of reference, where $\vec{B}$ is a local magnetic field vector. The resulting $\pi\left(\Delta m_{l}=0\right)$ and $\sigma\left(\Delta m_{l}= \pm 1\right)$ spectral lines of the Stark multiplet are polarized parallel and perpendicular to the electric field direction, respectively. Here, $\Delta m_{l}$ is the variation of magnetic orbital momentum. Therefore, the polarization of the observed lines is sensitive as to the orientation of the vector $\vec{E}_{L}$ but also to the direction of the vector of magnetic field $\vec{B}$ in the plasma.

Employing polarization measurements from the central unshifted $\sigma_{0}$ line, it is possible to reconstruct the pitch angle of the magnetic field by the MSE polarimetry system. ${ }^{1-3}$ In spectral MSE measurements, the line splitting,

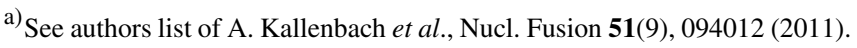

$\Delta \lambda$, depends on $\left|\vec{E}_{L}\right|$, and therefore it allows us to measure $|\vec{B}|{ }^{4-6}$

The MSE diagnostic is routinely used as a tool to improve the equilibrium reconstruction..$^{7-11}$ However, the desired high precision for magnetic field measurement could not be achieved due to a number of inaccuracies in earlier analysis such as the treatment for the population densities of excited magnetic sub-levels. ${ }^{12}$ The situation was improved significantly in the last years. Using new collisional radiative models, ${ }^{13,14}$ one resolved finally the discrepancy between the measured line ratio within the $\sigma$ and $\pi$ polarization fraction in the MSE spectra for JET and ALCATORC Mod. ${ }^{15,16}$ The data from the non-Local Thermodynamic Equilibrium (non-LTE) model predict even much stronger deviation from statistical expectation for MSE line intensities at ITER conditions in comparison with the present devices. ${ }^{17}$ However, the Zeeman effect was often neglected in the beam emission analysis with regard to its smallness compared to the Stark effect. ${ }^{12}$ The impact of the Zeeman effect on the MSE spectra was considered either in the MSE polarimeter measurements at ALCATOR ${ }^{12}$ or it was envisaged to implement the Zeeman effect in the ab initio modeling. ${ }^{18}$

In this paper, the effect of magnetic and electric fields on the Balmer- $\alpha$ emission is revisited. The atomic physics of the combined Zeeman-Stark effect ${ }^{19-23}$ is adapted for the application in MSE measurements, and the Zeeman effect and fine-structure are discussed in view of the spectral MSE observations. The model is prepared for even more 
refinements which can be done in future, e.g., by including contributions of radial electric fields. The recently developed MSE forward model ${ }^{24}$ is extended and takes the Zeeman-Stark effect and the spin-orbit coupling into account in order to describe the measured MSE spectra. Finally, the results of the measurements are compared with results from an equilibrium solver (CLISTE $)^{25}$ for ASDEX Upgrade experimental conditions.

\section{ATOMIC MODEL OF THE ZEEMAN-STARK MULTIPLET}

An atomic model of hydrogen atom in the presence of electromagnetic field represents a topic that is still far from being closed, specially in studies of high Rydberg states or in the case of strong fields, ${ }^{26}$ although the experimental data for the simplest configurations are understood now. So, for instance, it is an established fact that the Zeeman effect, or more precisely the Paschen-Back effect, dominates the finestructure splitting of the Balmer- $\alpha$ line emission at the plasma edge of fusion devices. ${ }^{27}$ In the case of Maxwellian distribution function of atoms, every magnetic component of the spectral line is described using a Doppler profile taking into account the different source of excited atoms. ${ }^{27,28}$ In the case of MSE measurements, the emissions take place predominantly in the static crossed electric and magnetic fields, being a subject of studies for high Rydberg states. ${ }^{26,29}$ The influence of the fields onto the emission pattern of the Balmer- $\alpha$ line in fusion plasmas was shown in Refs. 19 and 21 and for MSE observations in Ref. 30. The energy displacement of the levels caused by the magnetic field depends on the mutual orientation between the vectors $\vec{E}_{L}$ and $\vec{B} \cdot{ }^{26}$ In the first order perturbation theory, the linear and quadratic dependence on the strength of magnetic field appears in the energy expression if vectors are orthogonal to each other ${ }^{31}$

$$
E^{ \pm}(n, k) \approx \pm \Omega+k \sqrt{\left(\frac{3}{2} n F\right)^{2}+\Omega^{2}}
$$

Here, $E^{ \pm}(n, k)$ is the energy ${ }^{32}$ of levels with $n=n_{1}+n_{2}+\left|m_{l}\right|$ +1 , where $n$ is the principal quantum number, $m_{l}$ is the orbital magnetic number, $k=n_{1}-n_{2}$ is the electric quantum number, and integers $n_{1}$ and $n_{2}$ are the parabolic quantum numbers, with $0 \leq n_{1}<n$ and $0 \leq n_{2}<n$. The parameter $\Omega=1 / 2 \cdot B / B_{0}$ is the magnetic field strength $\left(B_{0}=2.35 \cdot 10^{5} \mathrm{~T}\right)$ and $F=E_{L} / E_{0}$ is the electric field strength $\left(E_{0}=5.142 \cdot 10^{11} \mathrm{~V} / \mathrm{m}\right)$. Expression (1) is valid only if $F, \Omega \gg \delta$, where $\delta$ is the fine-structure splitting. Two effects caused by the magnetic field are observed from expression (1). First, the magnetic field efficiently increases the electric field strength of the pure parabolic states. Second, the linear term removes their double degeneracy due to the interaction of spin magnetic moment with the magnetic field. In the case of MSE observation, the ratio between the electric and magnetic fields remains constant $\left(\vec{E}_{L}=\vec{v} \times \vec{B}\right)$ and as in majority of cases $F>\Omega$, expression (1) reduces to

$$
E^{ \pm}(n, k) \approx \frac{3}{2} n F\left(k \pm \zeta_{n}+\frac{k}{2} \zeta_{n}^{2}\right),
$$

with

$$
\begin{aligned}
\zeta_{n} & =\frac{\Omega}{3 / 2 n F} \\
& =\frac{2}{3 n} \cdot \frac{v_{0}}{v \sin \omega},
\end{aligned}
$$

considering only one term of expansion in $\Omega /(3 / 2 n F)$. Here, $v_{0}=2.188 \cdot 10^{6} \mathrm{~m} / \mathrm{s}$ is the atomic unit of velocity, $v=\sqrt{2 E / m}$ is the velocity of beam atom in $\mathrm{m} / \mathrm{s}$, and $\omega$ is the angle between vectors $\vec{B}$ and $\vec{v}$. Parameter $\zeta_{n}$ characterizes the impact of the magnetic field on the displacement of energy levels for MSE observations. Similar to the contribution of the magnetic field in the final expression for energy, one could also estimate the relative contribution of the fine-structure splitting relative to the Stark effect. ${ }^{33}$ In this case,

$$
\zeta_{n}^{f_{s}} \approx \frac{\alpha^{2}}{n^{3}} \cdot \frac{2}{3 n F},
$$

where $\alpha=1 / 137$ is the fine-structure constant. Substituting the energy of beam atoms on the order of $10 \mathrm{keV} / \mathrm{amu}$ and magnetic field of $2 \mathrm{~T}$, which corresponds to the condition of third energy components at ASDEX Upgrade, one obtains for the levels of $n=3$, the values $\zeta_{3}=0.35$ and $\zeta_{3}^{f_{s}}=0.08$, and for levels of $n=2$, the values $\zeta_{2}=0.52$ and $\zeta_{2}^{f s}=0.4$. Obviously, the magnetic field and the fine-structure splitting could not be neglected in the description of the MSE spectra at these low atomic energies. The impact of these effects is different for the MSE spectrum. The fine-structure splitting of $n=2$ shifts the transition of the $\mathrm{D}_{\alpha}$ line as a whole. In contrast, the magnetic field affects both the line splitting and purity of the new states. The second effect leads to $\sigma$ - and $\pi$-transitions containing different polarization fractions. This fact plays a more important role in the MSE spectra analysis as depending on the observation geometry, the shift caused by the fine-structure alone could be negligibly small relative to the Doppler shift of the beam atoms. The general considerations shown above must be observed in the atomic data, e.g., energy levels and line intensities measured in crossed fields.

The calculation of atomic data in crossed static electric and magnetic fields was performed in the frame of the perturbation theory of the basis of the field-free wavefunctions in the reference frame as shown in Fig. 1. In this coordinate system, the Lorentz field $\overrightarrow{E_{L}}=\vec{v} \times \vec{B}$ is taken to be parallel to the $z$-axis, and the vector of magnetic field $[\vec{B}=(B, 0,0)]$ is aligned along the $x$-axis. The vector of the velocity $\vec{v}$ is depicted to be in the $x-y$ plane $[\vec{v}=(0,-v, 0)]$. The direction of observation is shown by the vector $\vec{s}$ with the polar angle $\phi$ and the azimuthal angle $\theta$. The plane normal to the vector $\vec{s}$ defines the direction of the orthogonal polarization vectors $\vec{e}_{1}$ and $\vec{e}_{2}$ so that $\vec{e}_{1} \cdot \vec{e}_{2}=0$. In addition, we choose the vector $\vec{e}_{2}$ to be parallel to the xy plane. The energies of the new eigenstates in crossed fields, as shown in Fig. 1, were obtained by diagonalizing the Hamiltonian of the atom. The latter includes the relativistic effects, fine-structure splitting, and operators of interaction of the atom with electric and magnetic fields. We note that the Lamb-shift being on the order of $0.0353 \mathrm{~cm}^{-1}$ for $n=2$ levels compared to $0.365 \mathrm{~cm}^{-1}$ of fine-structure separation was not included in our calculations. The details of calculations in crossed fields could be found elsewhere. ${ }^{21}$ In all cases, the results reproduced well 


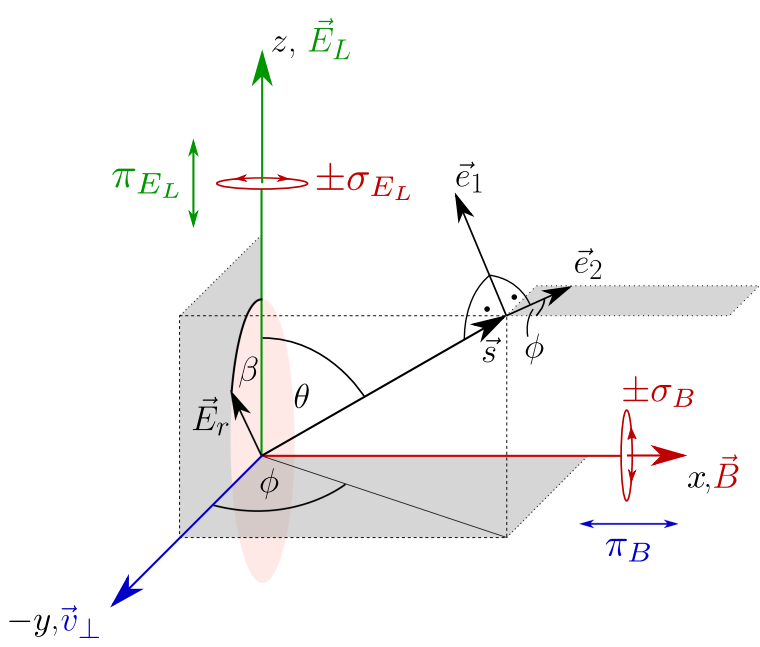

FIG. 1. Frame of reference and vector orientation used in the present calculation: $\vec{B}$ is the vector of magnetic field, $\vec{E}_{L}$ is the vector of induced Lorentz field, $\vec{v}_{\perp}$ is the vector of atom velocity, $\vec{s}$ denotes the direction of observation, $\vec{e}_{1}, \vec{e}_{2}$ are the polarization vectors, and $\phi$ and $\theta$ are the angles determining the observation orientation. The electric field induces linear polarized emission in the direction parallel to $\vec{E}_{L}\left(\boldsymbol{\pi}_{\mathbf{E}_{\mathbf{L}}}\right)$, circular polarized emission perpendicular to $\vec{E}_{L}\left(\sigma_{\mathbf{E}_{\mathbf{L}}}\right)$; the magnetic field induces linear polarized emission in the direction parallel to $\vec{B}\left(\boldsymbol{\pi}_{\mathbf{B}}\right)$ and circular polarized emission perpendicular to $\vec{B}\left(\sigma_{\mathbf{B}}\right)$.

the cases of pure Zeeman and Stark effects. In Fig. 2, we show the example of calculation of $n=2$ energy levels in crossed fields, whereas in the case of MSE measurements, the ratio between the strength of electric and magnetic fields was kept constant. Figure 2(a) shows the behavior of the levels for the weak field conditions (Ref. 34, pp. 239-242). These conditions are out of relevance for the parameters in fusion plasmas but they help to control the calculations. So, for instance, the quadratic Stark effect for the weak field could be well reproduced by switching off the magnetic field in the calculations (thin dashed lines). These levels are doubly degenerated. The presence of magnetic field removes the degeneracy of all the levels due to the spin of the atom. In the case of extremely weak field $(F, \Omega<<\delta)$, the behavior of the levels reproduces the properties of Zeeman and Stark effects as the splitting of the energy levels is proportional to the total angular momentum (Ref. 33, p. 154, Ref. 34, p. 240). By further increasing the field strength, two central unshifted components, separated on the order of $\delta$ in the case of pure Stark effect, start to show the linear dependence on the magnetic field for the fields on the order of $\delta / 2$ and higher. For outermost components, the dependence on the magnetic field appears even earlier. More detailed description of the level behavior could be performed by investigating the Hamiltonian in the general form. ${ }^{31}$ For the case of strong fields, Fig. 2(b) shows the dependence as observed in Eq. (1). Here, it is possible to separate the angular momentum in the spin and orbital part. The orbital momentum interacts with the electric field resulting in parabolic states $\left(m_{l}=0, \pm 1,0\right)$ shown as thin dashed lines.

One also observes the offset of two central components due to the fine-structure separation. Again as in the case of weak field, these levels are doubly degenerated. The interaction of the magnetic field with orbital momentum, e.g., the quadratic term in Eq. (2), increases the displacement in energy of the new states as shown by dashed-dotted lines for two outermost components. Finally, the interaction of the magnetic field with spin momentum splits every levels (dashed-dotted lines) into two $\pm \Omega$ components relatively to the Stark states (solid lines). In Fig. 3, we show the results of calculations for the experimental conditions relevant in fusion plasmas for the intensity of the $H_{\alpha}$ line. First, we consider the case without magnetic field as shown in Fig. 3(a). In the pure MSE case with $\vec{E}_{L}$ pointing to the $z$-direction, the pattern consists of fifteen lines with equidistant line splitting, nine of which are, in practice, detectable. The individual transition lines are
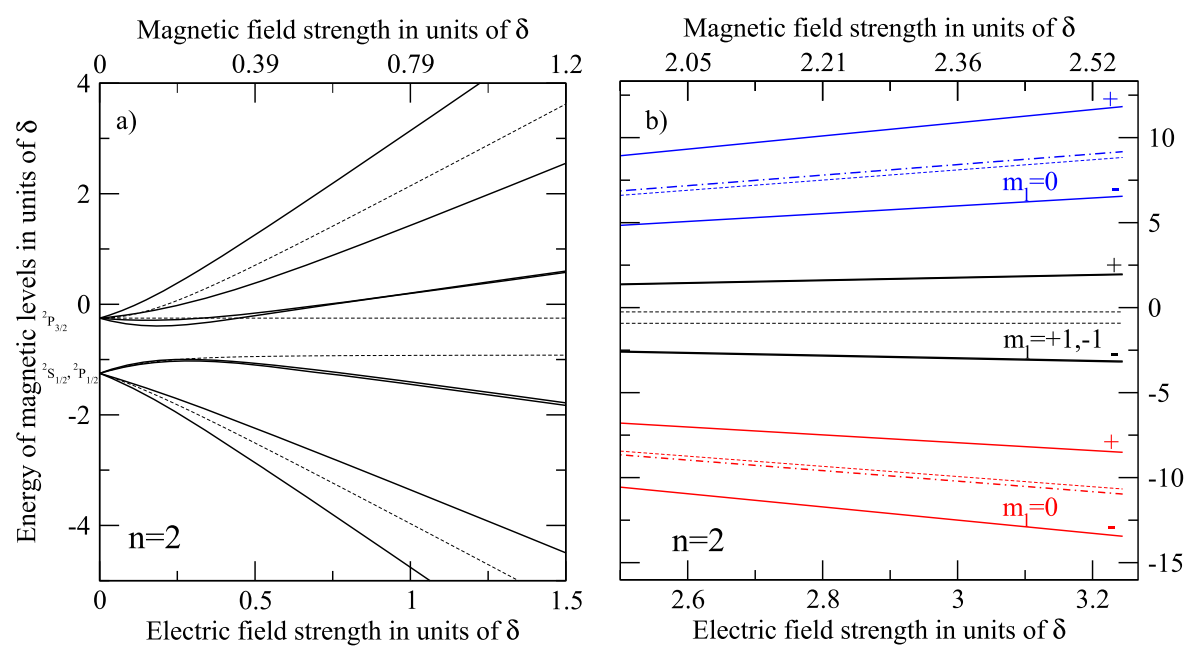

FIG. 2. Energy levels of $n=2$ in crossed electric and magnetic fields. The energy, strength of magnetic, and electric field are shown in units of the field-free splitting $\delta=0.365 \mathrm{~cm}^{-1}$ between $j=3 / 2$ and $j=1 / 2$ levels. The zero in the ordinate corresponds to the non-relativistic energy of the $n=2$ levels so that $E(j=3 / 2) / \delta=-1 / 4$ and $E(j=1 / 2) / \delta=-5 / 4$. The ratio $\Omega / F=0.79$ (the beam energy is $10 \mathrm{keV} / \mathrm{amu}$ and the magnetic field is $2 \mathrm{~T}$ ) is kept constant in the calculation. (a) Case of weak electric and magnetic fields: $F, \Omega \approx \delta$. Thin dashed lines show the energy of the levels for the Stark effect only $(\Omega=0$ ); solid lines correspond to the calculation of the Zeeman-Stark effect. (b) Case of strong fields: $F, \Omega \gg \delta$, where different colors correspond to states with different $m_{l}$ numbers (parabolic states). Dashed-dotted lines for the outermost components show the Zeeman-Stark effect calculations neglecting the spin of the atoms; for other lines, the notation is the same as in (a). 

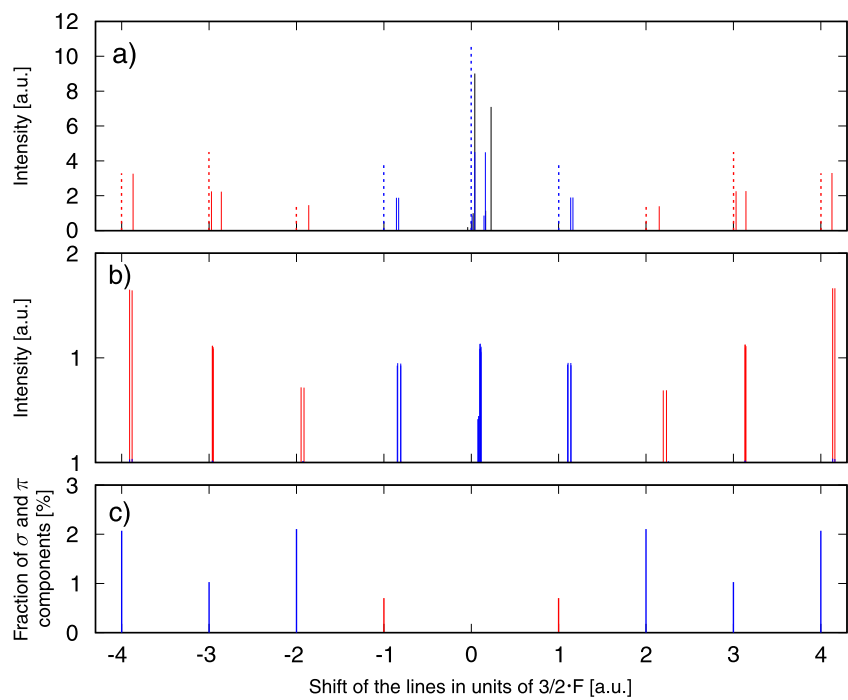

FIG. 3. Calculation of $D_{\alpha}$ multiplet for the beam energy of $10 \mathrm{keV} / \mathrm{amu}$ and magnetic field of $2 \mathrm{~T}$. Shift of the polarization components is shown in the units of $3 / 2 F$ (a.u.), blue lines denote the $\sigma$ components, and red lines denote the $\pi$ components. Fine-structure field-free calculations are shown as black thin lines to indicate the scale of splitting. (a) Stark effect calculations: solid lines show the results of calculation taking the fine structure into account; dashed lines with dots are the Stark effect calculation in a strong field, e.g., fine-structure and relativistic effects are neglected. (b) Solid lines show the results of Zeeman-Stark effect calculations and dashed lines show the results of calculations in strong fields as in (a). (c) Fraction of $\sigma$ components at $\pi$ Stark lines and $\pi$ components at $\sigma$ Stark lines due to the Zeeman effect. Here, the intensity of all lines in vicinity of corresponding transition was summed up.

perpendicularly polarized $(\sigma)$ or parallelly polarized ( $\pi$ ) to $\vec{E}_{L}$ components. For each polarization state, the sum over all lines, including weak ones, is conserved so that $\sum_{i j}^{\pi} I^{\pi}=1 / 2 \sum_{i j}^{\sigma} I^{\sigma}$. The relative intensities calculated with this approach (dashed lines) agree with calculations (Refs. 35 and 34, p. 277) and also field-free (thin solid lines) line strengths, $\sum_{i j}^{\pi} I^{\pi}=36.907$ a.u. ${ }^{36}$ By including the fine-structure in the calculations, one shifts the energy of the whole multiplet and splits the components with final states $m_{l}= \pm 1$ according to the results of Fig. 2 . In Fig. 3(b), one observes the impact of magnetic field on the multiplet. By neglecting the spin of the atom, one observes the same picture as in the case of pure MSE but the line positions are shifted due to the quadratic term in Eq. (2). This shift is less than the corresponding displacement induced by the finestructure as discussed before (dashed lines). By taking the spin of the atom and fine-structure into account, one observes the splitting of the components due to the linear term of interaction (solid lines). The following consequence for the MSE diagnostic can be observed. One detects the redistribution of the polarization pattern, e.g., the pure Stark $\pi$ transitions obtain the small fraction of the $\sigma$ contribution, and on the other hand, the pure Stark $\sigma$ transitions obtain certain fraction of $\pi$ components. In all cases, the sum over all $\sigma$ and $\pi$ components remains constant, although the different polarizations appear at the same positions compared to the Stark effect. In order to exemplify this effect, we show the fraction of $\sigma$ components at Stark- $\pi$ lines and $\pi$ fraction at Stark- $\sigma$ lines in Fig. 3(c). One observes the mixing on the order of 1\%-3\% due to the Zeeman effect. The strongest mixing of polarization is observed at $\pi_{4}$ and $\pi_{2}$ lines. The fraction is shown at the position of Stark lines, although the emission takes place at slightly different positions as shown in (b). Thus, the aim of this paper is to analyze the impact of the mixing of polarization components and of the line shift to the experimental data and, moreover, to determine their effect on the pitch angle and on the magnetic field, respectively.

\section{COMPARISON WITH EXPERIMENTAL DATA FROM ASDEX UPGRADE}

\section{A. Measurement technique}

We now give a brief description of the setup of the spectroscopic diagnostic at ASDEX Upgrade, which is described in detail in Ref. 24. Similar systems can be found in many fusion experiments..$^{30,37-39}$ In ASDEX Upgrade, the beam emission of six different positions along the beam axis (position) is observed with a mirror, installed near the plasma boundary. A lens system is used to focus the light onto a fibre bundle, which relays the light to a spectrometer. Since the fibres are arranged in one vertical line at the entrance slit of the spectrometer, a two-dimensional CCD-camera is used to record the full beam emission spectra, including the intense Balmer$\alpha$ edge emission, for each radial position. To avoid saturation on the CCD-chip, the edge emission line is blocked out by a thin metal wire which is positioned at the exit plane of the spectrometer exactly at the wavelength of this line.

\section{B. Observed spectrum}

A typical beam emission spectrum observed at ASDEX Upgrade is shown in the upper plot in Fig. 4 for one position

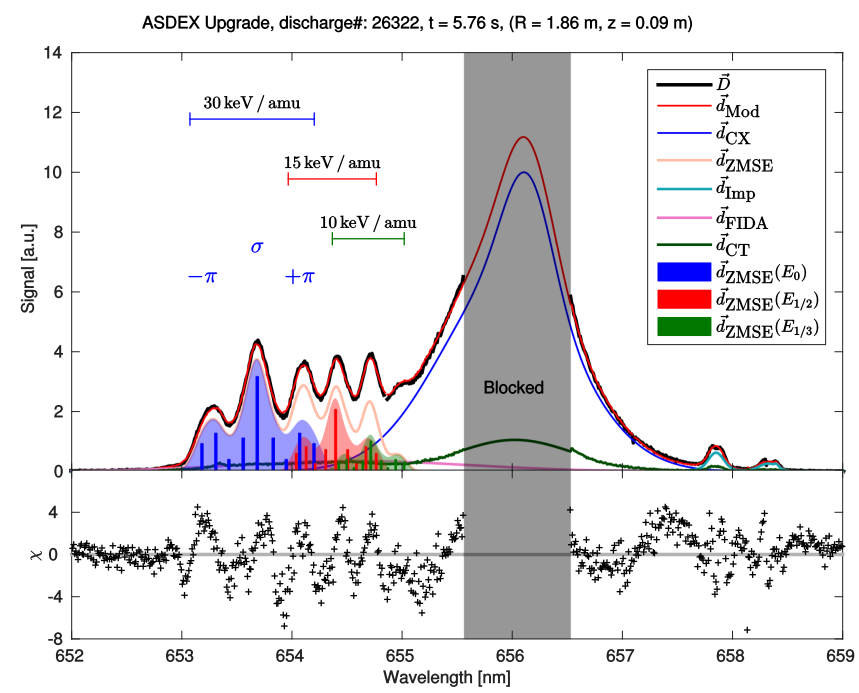

FIG. 4. Top plot: Experimental data from the ASDEX Upgrade beam emission spectrum $\vec{D}$, modeled spectrum $\vec{d}_{\text {Mod }}$, consisting of active and passive charge-exchange emission $\vec{d}_{\mathrm{CX}}$, the combined Zeeman and motional Stark effect and fine-structure multiplets $\vec{d}_{\text {ZMSE }}$, CII edge emission $\vec{d}_{\text {Imp }}$, fast ion $\mathrm{D}_{\alpha}$ component $\vec{d}_{\mathrm{FIDA}}$, and cross talk $\vec{d}_{\mathrm{CT}}$. The filled area represents the calculated ZMSE spectra for the full (blue), half (red), and third (green) energy components. In this measurement, the Balmer- $\alpha$ edge emission has been optically blocked to avoid over-exposure of the CCD detector. Both the experimental and the fitted data are background subtracted. Bottom plot: $\mathcal{X}$ as a measure for goodness of fit. 
$(R=1.86 \mathrm{~m}, z=0.09 \mathrm{~m})$ at $t=5.76 \mathrm{~s}$. The corresponding fitted data of the forward model are given in red. The dominating CX emission line is slightly shifted with respect to the cold $\mathrm{H}_{\alpha}$ and $\mathrm{D}_{\alpha}$ emission lines (at around $656.1 \mathrm{~nm}$ and $656.3 \mathrm{~nm}$ ). The gray rectangle indicates the spectral region at which the signal was suppressed by a blocking wire to avoid saturation at the CCD detector. On the blue-wing side $(653 \ldots 655 \mathrm{~nm})$, a Balmer- $\alpha$ splitting is clearly visible. It consists of a superposition of three Zeeman, Stark and fine structure (ZMSE) multiplets corresponding to the full, half, and third beam energies, each of them Doppler-shifted by $\Delta \lambda_{D}$ and overlapping. These are denoted as $\vec{d}_{\mathrm{ZMSE}}\left(\mathrm{E}_{0}\right), \vec{d}_{\mathrm{ZMSE}}\left(\mathrm{E}_{1 / 2}\right)$, and $\vec{d}_{\mathrm{ZMSE}}\left(\mathrm{E}_{1 / 3}\right)$.

Since the spectrum is overlapped partly by the CX emission line and completely by two flat and spectrally broad components (these being the fast ion $\mathrm{D}_{\alpha}$ emission line $\vec{d}_{\text {FIDA }}$ and the cross talk on the chip $\vec{d}_{\mathrm{CT}}$ ), a good description of these spectral features is required.

\section{Forward modeling of the combined Zeeman and motional Stark effect spectra}

Data analysis of the experimental data $\vec{D}$ is made by fitting a forward model resulting in synthetic data $\vec{d}$. The fit results in the best fitting values for the Lorentz field $\vec{E}_{L} \cdot{ }^{24,40,41}$

The forward model describing the measured data consists of a background signal $\left(d_{\mathrm{Bg}}\right)$, carbon impurity lines $\left(\vec{d}_{\mathrm{Imp}}\right)$, active charge exchange $\left(\vec{d}_{\mathrm{CX}}\right)$, a FIDA signal $\left(\vec{d}_{\mathrm{FIDA}}\right)$, and the ZMSE pattern $\left(\vec{d}_{\mathrm{ZMSE}}\right)$. Moreover, the cross talk on the CCD-chip during the readout process $\left(\vec{d}_{\mathrm{CT}}\right)$ is included in the forward model

$\vec{d}\left(\mathbf{F}^{E_{L}, B, \mathbf{L}-\mathbf{S}}, \vec{p}\right)=\vec{d}_{\mathrm{CX}}+\vec{d}_{\mathrm{Bg}}+\vec{d}_{\mathrm{Imp}}+\vec{d}_{\mathrm{CT}}+\vec{d}_{\mathrm{FIDA}}+\vec{d}_{\mathrm{ZMSE}}$,

where the parameter $\vec{p}$ reflects all settings, e.g., calibrations. Within the small range of wavelength, the background could be described by a constant. The charge exchange (CX) components (pedestal and active CX emission) were found to be well described by two overlapping Gaussian curves as functions of the wavelength. The widths of the Gaussians can be assigned depending on temperature and rotation velocity, which also affects the shift.

The impurity carbon lines are modeled in a similar fashion to the $\mathrm{D}_{\alpha}$-CX lines, using the temperature, carbon mass, line position, and amplitude.

The broad fast ion $\mathrm{D}_{\alpha}$ signal, $\vec{d}_{\text {FIDA }}$, overlaps the whole MSE spectrum but is of low intensity. ${ }^{42}$ In order to avoid the high modeling effort required for the small contribution of the FIDA signal, this component is approximated by two overlapping Gaussians of low heights at distinctly different wavelengths and with a large width of $\approx 1.5 \mathrm{~nm}$ (dependent on the position).

Since a frame transfer CCD-camera is used, smearing on the detector is generated during each frame transfer (vertical shift). This adds onto all the spectra on the CCD-chip and is considered in the model by $\vec{d}_{\mathrm{CT}}$.

The Balmer- $\alpha$ splitting is based on a MSE model which is extended by a correction factor that considers the line shift of the MSE lines due to the admixture of the Zeeman effect.
The extension of the forward model in Ref. 24 is to include the Zeeman effect and the effect of the spin-orbit coupling and relativistic effects in the description of the Balmer- $\alpha$ emission. This was done by extending the pure MSE model with correction factors for the wavelength splitting and for the intensity relation of the $\sigma$ and $\pi$-polarized Stark lines.

The model of the pure MSE spectrum considers all 15 $(\sigma$ and $\pi$ ) Stark components with a spectral profile function constructed by a Gaussian. To consider the different energies, three MSE spectra are modeled using the amplitude, $C_{\mathrm{b}_{i}}$, the Doppler shifted position of the central $\sigma_{0}$ line, the lines position, $\lambda_{E_{L i, \pi, \sigma}}$, and the line ratio $T_{P}$,

$$
\begin{aligned}
\vec{d}_{\mathrm{MSE}}=\sum_{i=1}^{3} C_{\mathrm{b}_{i}} & \left(T_{p} \sum_{\pi} A_{\pi} \exp \left[-\frac{1}{2}\left(\frac{\lambda-\lambda_{E_{L i, \pi}}}{\sigma_{w}}\right)^{2}\right]\right. \\
& \left.+\sum_{\sigma} A_{\sigma} \exp \left[-\frac{1}{2}\left(\frac{\lambda-\lambda_{E_{L i, \sigma}}}{\sigma_{w}}\right)^{2}\right]\right) .
\end{aligned}
$$

The fitting parameters are $C_{\mathrm{b}}, E_{L}, T_{p}$, the line shift, and the width, $\sigma_{w}$. Thus, for the modeling of one MSE multiplet, five free parameters were used. The Einstein coefficients $A_{\pi, \sigma}$ for the $\pi$ and $\sigma$ lines of the Stark spectrum are taken from Ref. 35. The width is mainly affected by the beam width and the instrument function. For the wavelength mapping, a quadratic dispersion relation was determined by three natural neon lines $\left(\lambda_{\mathrm{Ne} 1}=650.65 \mathrm{~nm}, \lambda_{\mathrm{Ne} 2}=653.29 \mathrm{~nm}\right.$, and $\left.\lambda_{\mathrm{Ne} 3}=659.90 \mathrm{~nm}\right)$.

Non-statistical distribution of sub-levels is considered by a density, magnetic field, and beam energy dependent parameter, $c_{n s}$, that was calculated by a collisional-radiative model ${ }^{13}$ and used as a correction factor for $T_{P}$,

$$
T_{P}^{n s}=c_{n s} \cdot T_{P} .
$$

The factor $c_{n s}$ is in the range of $0.8 \pm 0.04$ and needs to be considered in the later analysis.

In order to take into account the changes in the line ratio and the line mixing effect in the ZMSE case shown in Figs. 3 and 6 , a correction for the line ratio $T_{P}$ has to be done analogue to the statistical plasma correction in Eq. (8). Thus, the corrected line ratio is

$$
T_{P}^{n s, Z M S E}=c_{T_{P}} \cdot T_{P}^{n s} .
$$

To consider the line splitting of the ZMSE pattern in the forward model, the calculated splitting difference between the MSE-model and ZMSE-model is the implemented line dependent on the forward model

$$
\lambda_{\left(E_{L}, B\right)_{i, \pi, \sigma}}=\lambda_{E_{L i, \pi, \sigma}}+\Delta \lambda_{\left(E_{L}, B\right)_{i, \pi, \sigma}} .
$$

Thus the full description of the ZMSE pattern in the forward model is

$\vec{d}_{\text {ZMSE }}$

$$
\begin{gathered}
=\sum_{i=1}^{3} C_{\mathrm{b}_{i}}\left(T_{p} \sum_{\pi} A_{\pi} \exp \left[-\frac{1}{2}\left(\frac{\lambda-\left(\lambda_{E_{L i, \pi}}+\Delta \lambda_{\left(E_{L}, B\right)_{i, \pi, \sigma}}\right)}{\sigma_{w}}\right)^{2}\right]\right. \\
\left.+\sum_{\sigma} A_{\sigma} \exp \left[-\frac{1}{2}\left(\frac{\lambda-\left(\lambda_{E_{L i, \sigma}}+\Delta \lambda_{\left(E_{L}, B\right)_{i, \pi, \sigma}}\right)}{\sigma_{w}}\right)^{2}\right]\right) .
\end{gathered}
$$


Deviations of the beam direction and width between the three energy components in the applied MSE geometry are deduced from beam-into-gas calibration experiments. ${ }^{43}$ Thus separate widths and small deviations in positions can be calculated and incorporated into the forward model for each beam energy component, respectively. The model of the ZMSE spectrum considers all 15 ( $\sigma$ and $\pi$ ) Stark components with a spectral profile function constructed by a Gaussian. We note that the Gaussian shape of the magnetic lines used in the expression [Eq. (11)] represents only the approximation to the measured line profiles since the line shape is slightly asymmetric. The asymmetry differs for different $\pi$ - and $\sigma$-lines and could impact the interpretation of the data as shown in Refs. 18 and 43. The small deviations from Gaussian profile functions will be contemplated in future.

To consider the different energies, three MSE spectra are modeled using the amplitude, $A_{\mathrm{b}_{i}}$, the Doppler-shifted position of the central $\sigma_{0}$ line, the line position, $\lambda_{E_{L i, \pi, \sigma}}$, and the line ratio $T_{P}=\sum I_{\pi} / \sum I_{\sigma}$.

\section{Effect of atomic extension onto experimental quantities}

We now discuss the differences of the pure motional Stark effect and Zeeman-Stark effect (ZMSE) models for parameters relevant to the experimental results. In the case of the MSE model, we consider the simplest picture of strong field, neglecting the spin of the atom. For the given experimental conditions, Fig. 5(a) shows the modeled Doppler-shifted emission pattern for both calculations, MSE and ZMSE, normalized to their maximum value. For the magnetic field of $|B|=2.2 \mathrm{~T}$ and ASDEX Upgrade relevant beam energies, $E_{0}=29.8 \mathrm{keV} / \mathrm{amu}$, $E_{1 / 2}=14.9 \mathrm{keV} / \mathrm{amu}$, and $E_{1 / 3}=9.95 \mathrm{keV} / \mathrm{amu}$, one observes the pattern represented by the blue, red, and green curves. The MSE results are plotted using solid lines, and the ZMSE results are represented by dashed lines. The ZMSE pattern is

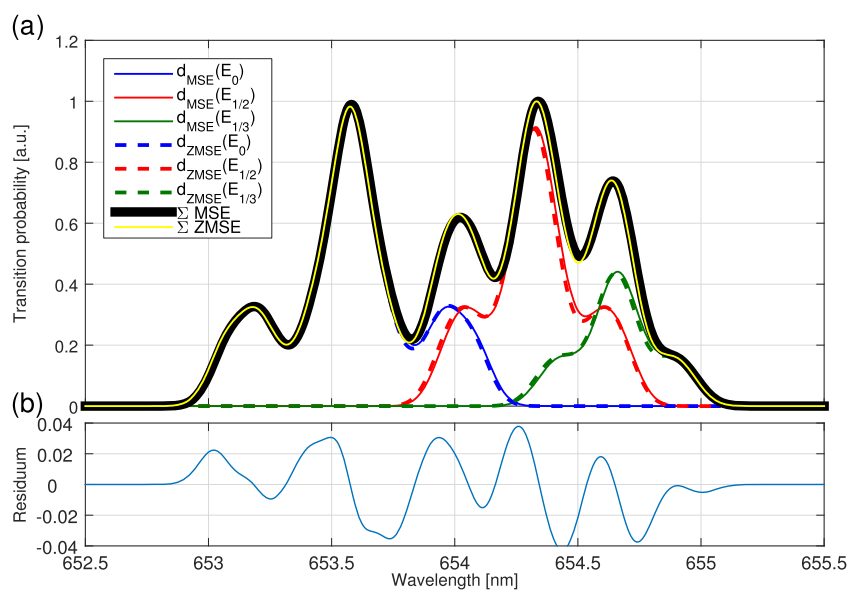

FIG. 5. (a) Doppler shifted beam profile for both MSE (black curve) and combined ZMSE (yellow curve). The MSE (solid lines) and ZMSE (dashed lines) for the individual ASDEX Upgrade beam energies are plotted in blue (full energy component, $E_{0}=29.8 \mathrm{keV} / \mathrm{amu}$ ), red (half energy component, $E_{1 / 2}$ $=14.9 \mathrm{keV} / \mathrm{amu}$ ), and green (third energy component, $E_{1 / 3}=9.95 \mathrm{keV} / \mathrm{amu}$ ). A typical ASDEX Upgrade magnetic field of $|\vec{B}|=2.3 \mathrm{~T}$ was applied. In (b), the residuum between both ZMSE-spectrum and MSE-spectrum is plotted. plotted in yellow and only slightly deviates from the MSE pattern (black). To reveal the spectral differences between both models, the residuum $I_{Z M S E}-I_{M S E}$ is plotted in Fig. 5(b). The obtained difference between both models is up to $4 \%$ with respect to the maximum intensity. The main cause for the big difference in the measured intensity is the shift of the line position. It is noted that the observed difference is strongly related to the chosen geometry setting $\left(\vec{E}_{L}, \vec{B}\right.$, and $\vec{s}$, cf. Fig. 1). For observation of the emission along $\vec{E}_{L}(\theta=\pi)$, all polarization directions perpendicular to $\vec{E}_{L}$ will be observed $\left(\pi_{B}, \sigma_{B}\right.$, and $\left.\sigma_{E_{L}}\right)$. At line-of-sight parallel to $\vec{B}(\theta=\pi$ and $\phi=\pi)$, all multiplet components which are perpendicularly polarized to $\vec{B}$ are observable $\left(\sigma_{B}, \sigma_{E_{L}}\right.$, and $\left.\pi_{E_{L}}\right)$.

In order to discuss the geometry dependence, Figs. 6(a) and 6(b) show the difference between MSE and ZMSE calculated spectra in dependence of the orientation of observation. Here the observation angles $\phi$ and $\theta$ are varied from $\phi=[0, \pi]$ and $\theta=[0, \pi / 2]$. The calculation was done for a beam energy of $E_{0}=30 \mathrm{keV} / \mathrm{amu}$ and the magnetic fields set to $2.3 \mathrm{~T}$.

For almost all observation angles, the Zeeman effect leads to an increase of the observed sum of $\pm \pi_{2}, \pm \pi_{3}, \pm \pi_{4}$ lines, and at the same time, a decrease of the observed $\pm \sigma_{1}, \sigma_{0}$ lines. The black box in Fig. 6 indicates the region of ASDEX Upgrade geometry. Here, the difference in the spectra results in about $0.35 \%$ at the position of the Stark $\pi$ component

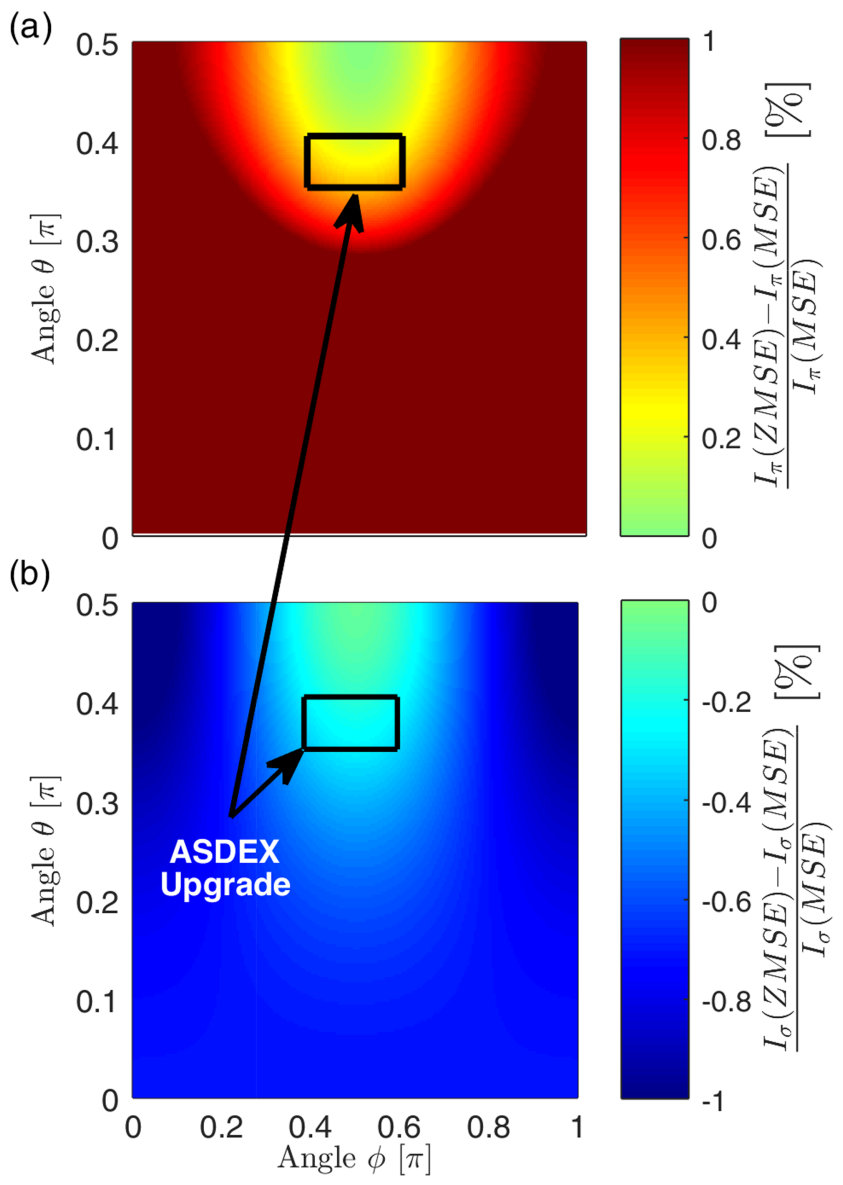

FIG. 6. Difference between pure MSE and ZMSE calculated signals $I_{\pi}$ (a) and $I_{\sigma}$ (b) normalized to its respective MSE calculated intensity in dependence of the geometry. The black boxes show the region of the ASDEX Upgrade geometry. The angles are given in values of $\pi$. 
and $-0.25 \%$ at the position of the Stark $\sigma$ component. The changes of the line intensities have impact on the observed line ratio $T_{p}=\sum\left(I_{\sigma}\right) / \sum\left(I_{\pi}\right)$, where the sum is extended over the $\pm \pi_{2}, \pm \pi_{3}, \pm \pi_{4}$ or $\pm \sigma_{1}, \sigma_{0}$ lines. This parameter is of crucial importance for the derivation of the pitch angle $\gamma$. The question is how this affects the pitch angle $\gamma$. We introduce the pitch angle, which measures the direction of the Lorentz field projected on the MSE geometry,

$$
\gamma=\arctan \frac{E_{L z}}{E_{L x}}
$$

The orientation of $\vec{E}_{L}$ is determined by the observation angle $\theta$ and the direction of the beam. The angle $\theta$ is a function of the observed line ratio

$$
\theta=\arccos \sqrt{\frac{1-T_{P}}{1+T_{P}}} .
$$

Figure 7 displays the changes in the pitch angle due to the extended atomic description of the forward modeled multiplet for the time point $t=5.01 \mathrm{~s}$ and for different radial positions of the ASDEX Upgrade discharge 26322. The black line indicates the correction made in the pitch angle when taking into account non-statistical distribution of the upper sub-levels. The correction in the pitch angle measurement is about $\Delta \gamma \approx 3.5^{\circ}$. Furthermore, the effect of the Zeeman effect is shown for three ASDEX Upgrade beam energies (blue, red, and green). For this case, the change in the pitch angle is about $\Delta \gamma_{Z M S E}=\left\{0.4^{\circ}, 0.7^{\circ}, 0.9^{\circ}\right\}$. It can be concluded that both corrections are quite significant compared to the required accuracy for fusion devices which is in the range of $0.1^{\circ} \ldots 0.5^{\circ} .4$ Thus the pitch angle reconstructions suffer systematically from a neglection of the Zeeman effect and from the assumption of statistical distribution of upper sub-levels. The total correction for the spectral MSE diagnostic is about $4^{\circ}$. However, the MSE diagnostic deriving the pitch angle from the polarization of the emission line is almost not affected by the effects described above. In fact, the non-LTE has no effect on the line emission polarization. Whereas the Zeeman effect introduces a circular

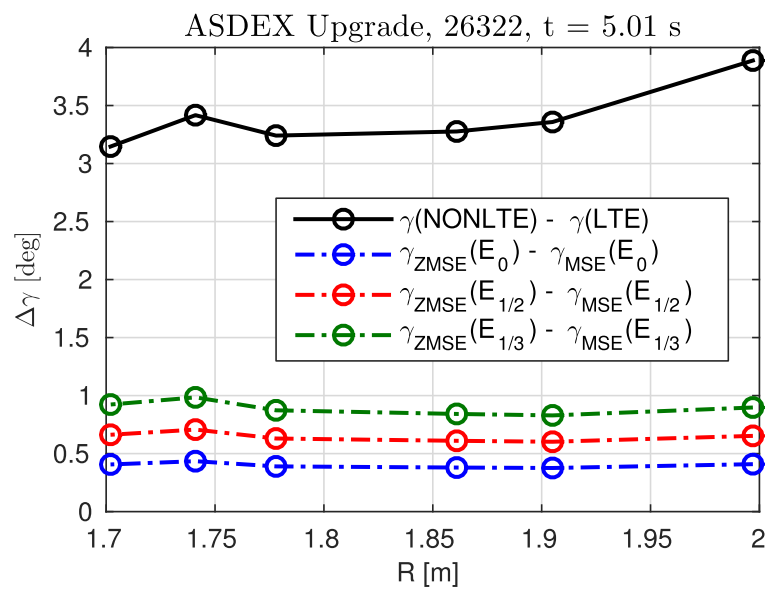

FIG. 7. Correction in the forward modeled pitch angle due to the extended atomic description of the Balmer- $\alpha$ spectrum: effect of non-statistical distribution of the upper sub-levels for the pure MSE case (black), effect of spectral ZMSE, including fine-structure and relativistic effects (red). The colors indicate the certain ASDEX Upgrade beam energy. polarization fraction on the emission lines. This circular polarization fraction reduces the useful linear polarization fraction but does not change the linear polarization angle.

As shown in Sec. II, the Zeeman effect and the finestructure cause a shift of the multiplet and a change in the line splitting. For ASDEX Upgrade relevant conditions, the multiplet is shifted by about $5 \%$ for $30 \mathrm{keV} / \mathrm{amu}$ to $11 \%$ for $30 \mathrm{keV} / \mathrm{amu}$ beam energies with respect to the $\sigma_{0}$-Stark line. The line splitting changes in the range of $1 \%(30 \mathrm{keV} / \mathrm{amu})$ to $2 \%$ (30 keV/amu). In Fig. 8 , the change in $|\vec{B}|$ due to the difference of the line splitting between the pure MSE case and ZMSE case is shown for varying splitting and ASDEX Upgrade beam energies. The splitting is the mean value taken from most intensive lines $(-4 \pi \ldots+4 \pi)$. The scattered symbols denote the experimental data taken from a magnetic field ramp-down discharge (\#26322); the inclined lines represent the fit referred to the experimental data. The color code corresponds to the beam energies. For a magnetic field of about $2.3 \mathrm{~T}$, a difference of $1.6 \%\left(E_{0}\right) \ldots 2.5 \%\left(E_{1 / 3}\right)$ can be seen. This is a significant effect and needs to be considered for the calculation of the absolute value of $B$. The aforementioned formulation of the ZMSE case with the spin-orbit coupling and relativistic effects is now included in the forward model, and the measured spectral MSE data, $\vec{d}$, at the ASDEX Upgrade are fitted using the forward model. ${ }^{24}$

\section{E. Validation of the ZMSE diagnostic}

In order to validate the forward model, a reference discharge has been conducted on the ASDEX Upgrade. The discharge parameter was chosen to reflect conditions that have been analysed with the CLISTE equilibrium code. ${ }^{25,44}$ Figure 9 shows the time evolution of the discharge indicating

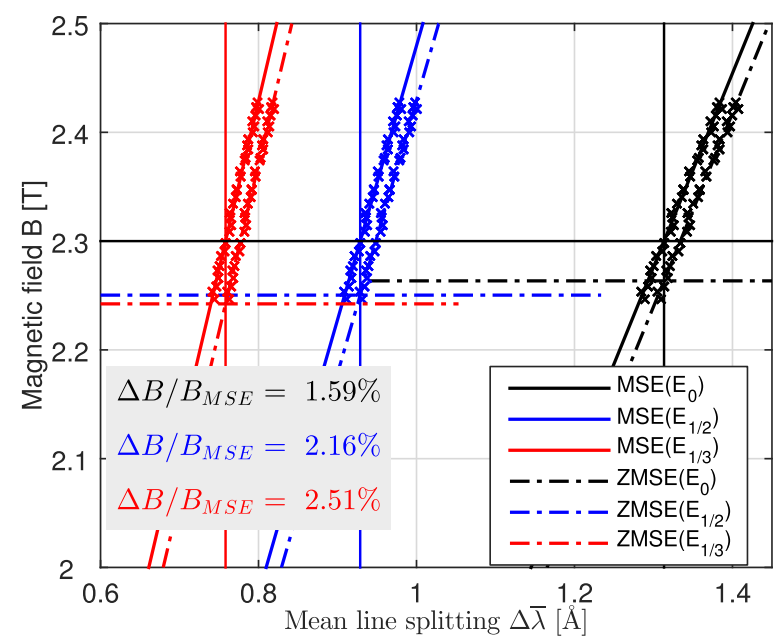

FIG. 8. Magnetic field variation as a function of the line splitting at the radial position $R=1.78 \mathrm{~m}$. The crosses represent the pure MSE case (along the solid lines) and ZMSE with fine-structure and relativistic effects (along the dashed-dotted lines) calculated splitting corresponding to a magnetic field ramp performed during ASDEX Upgrade discharge 26322. The lines along the experimental data represent a fit to these data. The horizontal black line indicates a magnetic field calculated with CLISTE corresponding to a MSE splitting value (vertical lines). The dashed horizontal lines represent the magnetic field values corresponding to the ZMSE model evaluated splitting value (vertical lines). The data are represented in color-codes for the three beam energies full (black), half (blue), and third (red). 
(a)

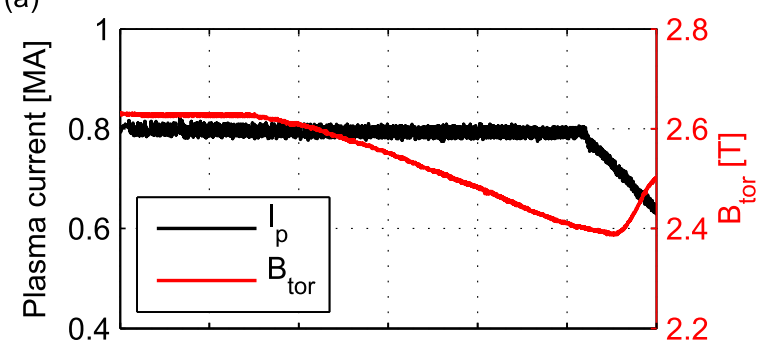

(b)

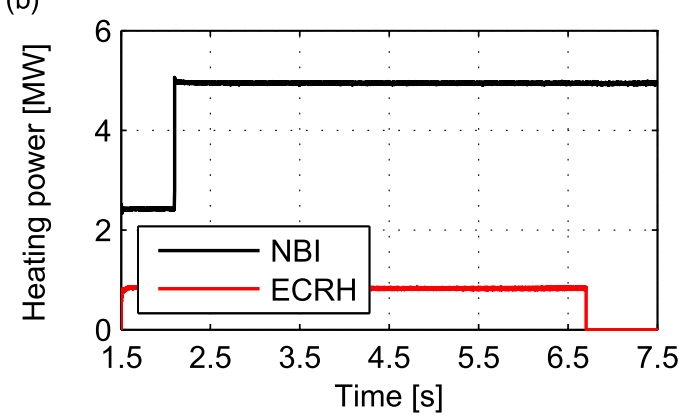

FIG. 9. Reference discharge on ASDEX Upgrade (\#26322): (a) Time traces of the toroidal magnetic field (red) and the plasma current (black); (b) applied plasma heating consisting of NBI (black) and ECRH (red) heating power.

a stationary plasma current of $I_{p}=0.8 \mathrm{MA}$ (a) and a stationary heating of $P=5.8 \mathrm{MW}$ (b) but a decrease in the absolute value of the toroidal magnetic field from $\left|B_{t o r}\right|=2.6 \mathrm{~T}$ to $\left|B_{t o r}\right|=2.4$ $\mathrm{T}$ (a). $B_{t o r}$ has been decreased by lowering the toroidal field coil current. If the forward model is correct, then the temporal evolution of the Lorentz field $\vec{E}_{L}=\vec{v} \times \vec{B}$ should show the same variation as the applied magnetic field and should agree with the independent analysis of the CLISTE equilibrium code.

Figure 10 shows the temporal evolution of the Lorentz field $\vec{E}_{L}=\vec{v} \times \vec{B}$ from both an independent analysis of CLISTE (blue) and from the fitted data of the forward model (red line) for two chosen positions.

The CLISTE data are directly derived from $\vec{E}_{L}^{C L}=\vec{v}_{\perp}$ $\times \vec{B}^{C L}$, where $\vec{v}_{\perp}$ is taken from calibration measurements of the beam and MSE geometry and $\vec{B}^{C L}$ is a result of the solution

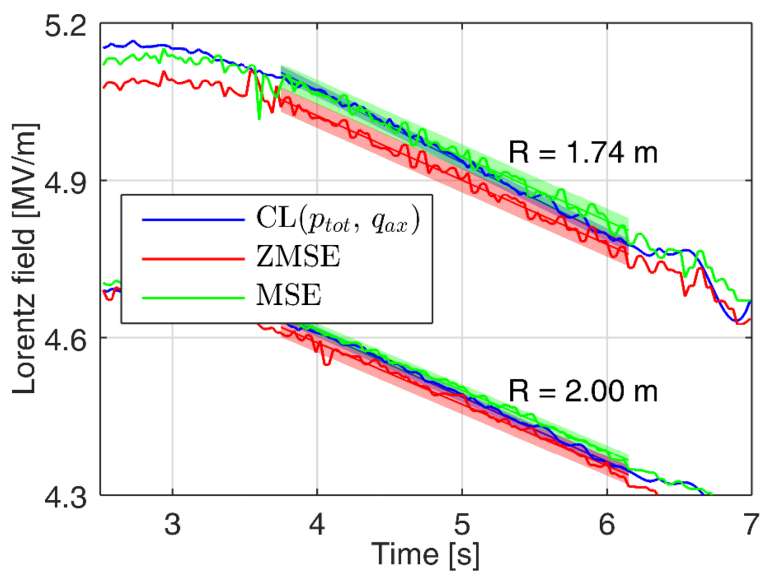

FIG. 10. Discharge \#26322: Time traces of the Lorentz field calculated with the CLISTE equilibrium code with run\# 2364 (blue), with the ZMSE forward model (red), and with the MSE forward model (green): For all methods, the fit functions (straight lines) and the related rmse confidence intervals (shadowed regions) are given. of the Grad-Shafranov-equation ${ }^{45}$ in CLISTE. The forward modeled Lorentz fields are calculated with the SchwartzschildEpstein equation. ${ }^{35}$ The CLISTE calculations were constrained by magnetic measurements, $q$, and the total pressure $\left(p_{\text {tot }}\right.$ $\left.=p_{k i n}+p_{F I}\right)$. Since sawtooth activity has been observed, the safety factor was set $q=1$ at the axis. In fact, this is not exact but setting $q=1$ at the inversion radius $\left(\rho_{\theta} \approx 0.23\right)$ leads to almost the same results. The kinetic contribution of the total pressure, $p_{k i n}=k_{B} \cdot\left(n_{e} T_{e}+n_{i} T_{i}\right)$, was obtained from kinetic measurements and integrated data analysis (IDA) ${ }^{46}$ The fast ion pressure contribution, $p_{F I}$, was calculated with the transport code TRANSP. ${ }^{47}$

The linear ramp down phase of about $6 \%$ between $t=3.8$ $\mathrm{s}$ and $t=6.2 \mathrm{~s}$ was assumed to follow the linear decrease of $B_{t o r}$ and fitted by a linear model. The precision for each position was estimated from the sum of the squared residuals. The resulting $2 \sigma$ error intervals are represented by the shaded regions and are about the same order for CLISTE and forward model data. However, in contrast to the CLISTE data, the precision of the forward model data was found to be position dependent. With $\sigma=0.3 \%$, the error is the lowest at the outermost position and rises towards the plasma core with a maximum value of $\sigma$ $=0.6 \%$ for the innermost position. This can be explained by the beam attenuation which leads to a decreasing signal-to-noise level towards the plasma.

The results show a small radius dependent difference in the bias up to $2.5 \%$ and a good agreement for the temporal variation between both methods. In all cases, the derived Lorentz and magnetic fields for MSE case are higher as in the case of the ZMSE model which is in agreement with results of Sec. II (Fig. 3). Indeed, the magnetic field causes additional splitting of the components so that the weaker Lorentz electric field is now required to describe the measured spectra. The MSE data are found even in slightly better agreement with CLISTE calculations as ZMSE results. The total error in the variation of the Lorentz field is $\Delta E_{L} / E_{L 0} \approx 0.5 \%$. The reasons for the position dependent error could be as follows:

1. Error in the CLISTE results since CLISTE cannot take into account fast ion anisotropy.

2. Imperfections in the optics components in the MSE setup, e.g., by non-optimal adjustment of the detection components which consists of a spectrometer, an objective, and a CCD-chip. The MSE diagnostic is described in detail in Ref. 24.

3. Use of an improper profile function for the MSE lines: in the present work, a Gaussian profile was applied. However, this is not exact. Dux has shown in Ref. 43 that the MSE profile is asymmetric due to the variation of the magnetic field along the line-of-sight when it is crossing a beam with a certain width. The effect is the strongest in the innermost position.

It can be concluded that local variations in the magnetic fields of less $0.5 \%$ can be detected. Moreover, the spectral ZMSE diagnostic can be used for the measurement of absolute values of the local magnetics with a high accuracy of about $1 \%$ or even better. The measured values have a high precision between $0.3 \%$ and $0.6 \%$. To improve the consistency with CLISTE results in the measurement of the absolute 
values, the difference in the bias has to be minimized. This could be done by applying asymmetric MSE profile functions and by increasing the accuracy. However, the findings show that the application of the ZMSE forward model is a suitable tool to confirm and, moreover, to improve equilibrium reconstructions.

\section{SUMMARY AND OUTLOOK}

In this paper, the influence of the Zeeman effect was analysed for the measurements of MSE spectra at the ASDEXUpgrade tokamak. The contribution of these effects to the Balmer- $\alpha$ beam emission spectrum has been investigated systematically for different geometry, beam energy, and magnetic field strength. First, we analyze the results depending on the Zeeman effect only. It was found that under typical ASDEX Upgrade conditions, the line splitting is affected by the ZMSE in the range of $1 \%$ for $30 \mathrm{keV} / \mathrm{amu}$ to $2 \%$ for $10 \mathrm{keV} / \mathrm{amu}$ deuterium beam energies. The changes in the observed line ratio $\sum_{i} I_{\pi}^{i} / \sum_{j} I_{\sigma}^{j}$ with $i=\{ \pm 2, \pm 3, \pm 4\}$ and $j=\{ \pm 1,0\}$ due the Zeeman effect are up to $2 \%$ (10 keV/amu). The discrepancies for the energy dependent line splitting and line ratio were included into the new ZMSE forward model as a correction parameter. The resulting changes in the absolute value of the magnetic field are about $1.6 \%$ (30 keV/amu) to $2.5 \%$ (10 $\mathrm{keV} / \mathrm{amu}$ ) which is in the range of the para- and diamagnetism. The measurements of the pitch angle were performed at the ASDEX Upgrade using the line ratio technique, e.g., the measured intensity ratio of the $\sum I_{\sigma} / \sum I_{\pi}$ components was used to obtain this angle. The non-LTE population distribution leads to the variation of the angle on the order of $2^{\circ}$. By taking the Zeeman effect into account, the calculated pitch angles change about $0.7^{\circ}$ from the analysis based on the atomic models of the pure non-LTE Stark effect. The proposed approach differs from the standard polarimetry technique which is less sensitive to the non-LTE conditions for the excited levels of beams in the plasma. However, the uncertainty on the order of $1^{\circ}-2^{\circ}$ in the pitch angle exists also for this standard approach. ${ }^{18}$

From these findings, it can be concluded that the accurate modeling of the Zeeman-Stark effect is required to fulfill the needed accuracy for the determination of the magnetic field strength. We note that the present analysis was performed in the first-order perturbation theory only. Also the results of the non-statistical model in pure parabolic Stark states and the impact of the Zeeman effect on the line ratios were not taken self-consistently into account. We are going to improve this model in the near future.

The extended forward model was validated with an ASDEX Upgrade discharge. The applied linear decrease of the toroidal magnetic field of about $6 \%$ could be reconstructed by the forward ZMSE model. The calculated Lorentz fields show a position dependent offset of $\Delta E_{L 0} \approx 0 \%$ to $2.5 \%$ and a difference in the inclination of about $\Delta\left(\delta E_{L}\right) / E_{L 0} \approx 0.5 \%$ compared to Lorentz fields calculated with the equilibrium solver CLISTE. We could show that the ZMSE forward model leads to slightly lower Lorentz fields compared to the MSE forward model. This is consistent with results from the atomic physics calculations, which showed that the line splitting is increased by the Zeeman effect and the fine-structure. The high accuracy in both the absolute value and the time development demonstrates the spectral MSE diagnostic with the forward model of ZMSE to be a suitable tool for accurate equilibrium reconstruction. The error estimated from the statistical noise is slightly lower then the error of the CLISTE data for the outer positions but increases towards the inner positions due to beam attenuation.

Further improvements could be the reduction of the noise by improved hardware settings, e.g., not using the optical path of the polarimeter setup. Furthermore, the uncertainty of the data has shown the need of a full statistical description of the forward model, for example, by a Bayesian approach. Moreover, the forward model can be refined by considering additional electric field components, e.g., radial electric field.

The results advance the accuracy and precision of spectral motional Stark effect measurements. One application could be the investigation of magneto-hydrodynamic stability in the presence of fast ions which is a topic of high importance for the fusion project ITER.

\section{ACKNOWLEDGMENTS}

This work has been carried out within the framework of the EUROfusion Consortium and has received funding from the Euratom research and training programme 2014-2018 under Grant Agreement No. 633053. The views and opinions expressed herein do not necessarily reflect those of the European Commission. The authors would like to express their gratitude to the members of the ASDEX Upgrade Team and Andreas Dinklage for valuable discussions.

${ }^{1}$ F. Levinton, R. Fonck, G. Gammel, R. Kaita, H. Kugel, E. Powell, and D. Roberts, Phys. Rev. Lett. 63, 2060-2063 (1989).

${ }^{2}$ F. Levinton, G. Gammel, R. Kaita, H. Kugel, and D. Roberts, Rev. Sci. Instrum. 61, 2914-2919 (1990).

${ }^{3} \mathrm{~F}$. Levinton, in 9th Topical Conference on High Temperature Plasma Diagnostics, Santa Fe, NM, 15-19 March 1992 [Rev. Sci. Instrum. 63, 5157-5160 (1992)].

${ }^{4}$ R. Wolf, A. Bock, O. Ford, R. Reimer, A. Burckhart, A. Dinklage, J. Hobirk, J. Howard, M. Reich, and J. Stober, J. Instrum. 10, P10008 (2015).

${ }^{5}$ E. Foley, F. Levinton, H. Yuh, and L. Zakharov, Rev. Sci. Instrum. 79, 10F521 (2008).

${ }^{6}$ L. Zakharov, J. Lewandowski, E. Foley, F. Levinton, H. Yuh, V. Drozdov, and D. McDonald, Phys. Plasmas 15, 092503 (2008).

${ }^{7}$ S. Hirshman, D. Lee, F. Levinton, S. Batha, M. Okabayashi, and R. Wieland, Phys. Plasmas 1, 2277-2290 (1994).

${ }^{8}$ B. Stratton, D. Long, R. Palladino, and N. Hawkes, Rev. Sci. Instrum. 70, 898-901 (1999).

${ }^{9}$ R. Wolf, S. Günter, F. Leuterer, A. Peeters, G. Pereverzev, O. Gruber, M. Kaufmann, K. Lackner, M. Maraschek, P. McCarthy et al., Phys. Plasmas 7, 1839-1844 (2000)

${ }^{10}$ R. Wolf, J. Hobirk, G. Conway, O. Gruber, A. Gude, S. Günter, K. Kirov, B. Kurzan, F. Leuterer, M. Maraschek et al., Nucl. Fusion 41, 1259 (2001).

${ }^{11}$ C. Petty, W. Fox, T. Luce, M. Makowski, and T. Suzuki, Nucl. Fusion 42, 1124 (2002).

${ }^{12} \mathrm{H}$. Yuh, "The motional Stark effect diagnostic on Alcator C-Mod," Ph.D. thesis, Institute of Technology, Massachusetts, 2005, URL: http://hdl.handle.net/1721.1/34976.

${ }^{13}$ O. Marchuk, Y. Ralchenko, R. Janev, W. Biel, E. Delabie, and A. Urnov, J. Phys. B: At., Mol. Opt. Phys. 43, 011002 (2010).

${ }^{14}$ O. Marchuk, Y. Ralchenko, and D. Schultz, Plasma Phys. Controlled Fusion 54, 095010 (2012). 
${ }^{15}$ E. Delabie, M. Brix, C. Giroud, R. Jaspers, O. Marchuk, M. O’Mullane, Y. Ralchenko, E. Surrey, M. von Hellermann, K. Zastrow, and JET-EFDA Contributors, Plasma Phys. Controlled Fusion 52, 125008 (2010).

${ }^{16}$ I. Bespamyatnov, W. Rowan, K. Liao, O. Marchuk, Y. Ralchenko, and R. Granetz, Nucl. Fusion 53, 123010 (2013).

${ }^{17}$ Y. Ralchenko, O. Marchuk, W. Biel, T. Schlummer, D. Schultz, and E. Stambulchik, Rev. Sci. Instrum. 83, 10 D504 (2012).

${ }^{18}$ M. De Bock, N. Conway, M. Walsh, P. Carolan, and N. Hawkes, Rev. Sci. Instrum. 79, 10F524 (2008).

${ }^{19}$ R. Isler, Phys. Rev. A 14(3), 1015-1019 (1976).

${ }^{20}$ C. Breton, C. Demichelis, M. Finkenthal, and M. Mattioli, J. Phys. B: At. Mol. Phys. 13, 1703-1718 (1980).

${ }^{21}$ E. Souw and J. Uhlenbusch, Physica B+C 122, 353-374 (1983).

${ }^{22}$ W. Mandl, "Development of active Balmer-alpha spectroscopy at JET," Ph.D. thesis, Technische Universität München, 1991.

${ }^{23}$ E. Foley and F. Levinton, J. Phys. B: At., Mol. Opt. Phys. 39, 443 (2006).

${ }^{24}$ R. Reimer, A. Dinklage, R. Fischer, J. Hobirk, T. Löbhard, A. Mlynek, M. Reich, L. Sawyer, R. Wolf, and ASDEX Upgrade Team, Rev. Sci. Instrum. 84, 113503 (2013).

${ }^{25}$ P. Mc Carthy and ASDEX Upgrade Team, Plasma Phys. Controlled Fusion 54, 015010 (2012).

${ }^{26}$ V. Lisitsa, Atoms in Plasma (Springer Series, 1994), Vol. 14.

${ }^{27}$ J. Hey, C. Chu, and E. Hintz, J. Phys. B: At., Mol. Opt. Phys. 32, 3555-3573 (1999).

${ }^{28}$ V. Lisitsa, M. Kadomtsev, V. Kotov, V. Neverov, and V. Shurygin, Atoms 2, 195-206 (2014).

${ }^{29}$ P. Braun and E. Solovyev, J. Phys. B: At., Mol. Opt. Phys. 17, L211-L216 (1984).

${ }^{30}$ W. Mandl, R. Wolf, M. von Hellermann, and H. Summers, Plasma Phys. Controlled Fusion 35, 1373-1394 (1993).

${ }^{31}$ E. Stambulchik and Y. Maron, Phys. Rev. Lett. 113, 083002 (2014).
${ }^{32}$ The atomic units are used in this section.

${ }^{33}$ E. Condon and G. Shortley, The Theory of Atomic Spectra (Cambridge University Press, 1959).

${ }^{34} \mathrm{H}$. Bethe and E. Salpeter, Quantum Mechanics of One- and Two-Electron Atoms (Dover Publications, Mineola, New York, 2008).

${ }^{35}$ E. Schrödinger, Ann. Phys. 385, 437-490 (1926).

${ }^{36}$ L. Green, P. Rush, and C. Chandler, Astrophys. J., Suppl. Ser. 3, 37-50 (1957).

${ }^{37}$ R. Wolf, L. Eriksson, M. Von Hellermann, R. Konig, W. Mandl, and F. Porcelli, Nucl. Fusion 33, 1835-1847 (1993).

${ }^{38}$ K. McCarthy, N. Panadero, A. López-Fraguas, J. Hernández, and B. van Milligen, Contrib. Plasma Phys. 55, 459-469 (2015).

${ }^{39}$ N. Pablant, K. Burrell, R. Groebner, D. Kaplan, and C. Holcomb, Rev. Sci. Instrum. 79, 10F517 (2008).

${ }^{40}$ A. Dinklage, R. Reimer, R. Wolf, Wendelstein 7-X Team, M. Reich, and ASDEX Upgrade Team, Fusion Sci. Technol. 59, 406-417 (2011).

${ }^{41}$ R. Reimer, A. Dinklage, R. Wolf, M. Dunne, B. Geiger, J. Hobirk, M. Reich, ASDEX Upgrade Team, and P. J. McCarthy, Nucl. Fusion. 57, 046005 (2017).

${ }^{42}$ Y. Luo, W. Heidbrink, K. Burrell, D. Kaplan, and P. Gohil, Rev. Sci. Instrum. 78, 033505 (2007).

${ }^{43}$ R. Dux, B. Geiger, R. McDermott, T. Pütterich, E. Viezzer, and ASDEX Upgrade Team, "Impurity density determination using charge exchange and beam emission spectroscopy at ASDEX Upgrade," in 38th EPS Conference on Plasma Physics (European Physical Society, Strasbourg, 2011), URL: http://ocs.ciemat.es/EPS2011PAP/pdf/P1.056.pdf.

${ }^{44}$ P. Mc Carthy, Phys. Plasmas 6, 3554-3560 (1999).

${ }^{45}$ J. Wesson and J. Connor, Tokamaks (Clarendon Press, 1987).

${ }^{46}$ R. Fischer, C. Fuchs, B. Kurzan, W. Suttrop, E. Wolfrum, and ASDEX Upgrade Team, Fusion Sci. Technol. 58, 675-684 (2010).

${ }^{47}$ A. Pankin, G. Bateman, R. Budny, A. Kritz, D. McCune, A. Polevoi, and I. Voitsekhovitch, Comput. Phys. Commun. 164, 421-427 (2004). 